\title{
Adquisición de competencias transversales en alumnos de pregrado de Ciencias de la Salud en la Universidad Complutense: una experiencia positiva
}

\author{
L.A. Arráez-Aybara , J. Millán Núñez-Cortés a , D. Carabantes-Alarcón b' R. Lozano-Fernándezc, \\ I. Iglesias-Peinado c, E. Palacios-Alaizc, B. del Castillo-Garcíac, A. Nogales-Esperta
}

Introducción. Presentamos los resultados de la evaluación de las Jornadas Complutenses de Investigación para alumnos de pregrado en Ciencias de la Salud, una actividad académica con dos años de experiencia y una participación de 1.434 alumnos, creada en nuestra universidad para impulsar en los alumnos de pregrado habilidades comunicativas y de metodología en investigación. Sujetos y métodos. Mediante una encuesta anónima se evaluó en los alumnos el grado de satisfacción e interés (aprender metodología científica, aprender a hablar en público, etc.) y también en los moderadores de mesas (originalidad, planteamiento metodológico, presentación iconográfica, exposición oral, viabilidad y relevancia de las comunicaciones presentadas). Resultados. En las I Jornadas se inscribieron 500 alumnos, todos de la Universidad Complutense de Madrid (UCM), y se presentaron un total de 169 comunicaciones (129 orales, 40 pósters). En las II Jornadas lo hicieron 934 alumnos, 838 de la UCM y 96 de otras nueve universidades, y se presentaron un total de 286 comunicaciones (180 orales, 106 pósteres). Conclusiones. Es una actividad en la que el alumno pasa a ser un gestor activo y difusor de aprendizaje, y potencia de una manera dinámica habilidades en comunicación científica. También tiene beneficios para el profesorado y la institución universitaria, que la convierten en una 'terapia pedagógica holística'.

Palabras clave. Competencias transversales. Educación basada en competencias. Educación médica. Espacio Europeo de Educación Superior.

\section{Acquisition of general skills in undergraduate students in Health Sciences in the Complutense University: a positive experience}

Introduction. We present here the results of the appraisal of the Complutense Conference on Research for undergraduate students in Health Sciences, an academic activity which has been running for two years, with the participation of 1,434 students. It was set up in this University to encourage communication skills and research methodology among undergraduate students. Subjects and methods. By means of an anonymous survey, the levels of satisfaction and interest of the students were evaluated (learning scientific methodology, learning to speak in public, etc.); similarly, that of the panel moderators (originality, methodological approach, iconographic presentation, oral presentation, viability and relevance of presentations given). Results. The First Conference had 500 registered students, all from the Complutense University of Madrid (UCM), with a total of 169 presentations (129 oral, 40 poster). In the Second Conference there were 934 students, 838 from the UCM and 96 from nine other universities, with a total of 286 presentations (180 oral, poster 106). Conclusions. This is an activity in which the student becomes an active manager and imparter of learning, and dynamically reinforces his or her skills in science communication. It also has benefits for teachers and the University itself, making it a 'holistic pedagogical therapy'.

Key words. Competency-based education. Education medical. European Higher Education Area (EHEA). General skills.

'Todo hombre puede ser, si se lo propone, escultor de su propio cerebro S. RAMÓN Y CAJAL

\section{Introducción}

Pericia, aptitud, idoneidad para hacer algo o intervenir en un asunto determinado define, según el diccionario de la Real Academia Española (RAE) [1], la competencia. Por tanto, la compe- a Facultad de Medicina.

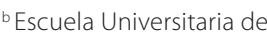
Enfermería, Fisioterapia y Podología. ' Facultad de Farmacia. Universidad Complutense de Madrid. Madrid, España.

\section{Correspondencia} Dr. Luis Alfonso Arráez Aybar. Facultad de Medicina. Universidad Complutense de Madrid. Avda. Complutense, s/n. E-28040 Madrid.

E-mail arraezla@med.ucm.es

El presente trabajo desarrolla la comunicación presentada el día 25 de octubre de 2007 en el XVIII Congreso de la Sociedad Española de Educación Médica, celebrado en Santa Cruz de Tenerife (Educ Med 2007; 10: 165)

Aceptación definitiva 29.07.2008 
tencia en general y la profesional en particular implica no sólo tener conocimientos (saberes) y saber aplicarlos (habilidades), sino también ser capaz de armonizar esas competencias (saberes y habilidades) con una actitud y valores adecuados al contexto social en que se desarrolla la profesión (saber cómo ser). Sin embargo, desde las últimas décadas del pasado siglo $\mathrm{xx}$, fue cada vez más notorio que los titulados universitarios en general y los sanitarios en particular, incluso con un buen expediente académico, no satisfacían esos parámetros.

Surge la necesidad de establecer modelos nuevos de enseñanza universitaria que consideren la formación de profesionales en términos de competencia y permitan una práctica profesional excelente. Uno de los elementos más dinamizadores de ese debate ha sido el proceso de convergencia hacia el Espacio Europeo de Enseñanza Superior (EEES), y se ha señalado su desarrollo como uno de los objetivos básicos que deben contemplar las próximas reformas de los planes de estudio tendentes a la armonización universitaria en la Unión Europea. En este contexto, en las titulaciones de Ciencias de la Salud, el modelo actual de currículo de pregrado tiene como meta alcanzar una conducta presidida por el profesionalismo y sus valores [2].

Estas nuevas orientaciones marcan la necesidad de precisar en cada titulación sus competencias [3]. Al hablar de competencias se distinguen dos tipos: competencias específicas, que caracterizan una profesión y la distinguen de otras, por lo que tradicionalmente han sido motivo de especial atención en la formación que lleva a las evaluaciones para la obtención del título académico que reconoce el ejercicio de la profesión, y competencias genéricas o transversales (CT), que engloban habilidades necesarias para ejercer eficazmente cualquier profesión y rebasan los límites de una disciplina para desarrollarse potencialmente en todas ellas.

\section{Competencias transversales}

La tabla I señala las CT propuestas por el proyecto 'Tunning' [4], con gran repercusión en el proceso de adaptación al EEES y que se resumen en:

- CT instrumentales: las más cognitivas son esenciales para el ejercicio de las profesiones sanitarias diversas. En la resolución de pro-
Tabla I. Competencias transversales propuestas en el proyecto 'Tunning' [4].

Instrumentales

- Capacidad de análisis y síntesis

- Capacidad de organización y planificación

- Conocimientos generales básicos

- Conocimientos básicos de la profesión

- Comunicación oral y escrita

- Conocimiento de idiomas

- Habilidades en el manejo de un ordenador

- Habilidades de gestión de la información

(buscar y analizar información de fuentes diversas)

- Resolución de problemas

- Toma de decisiones

Interpersonales

- Capacidad crítica y autocrítica

- Trabajo en equipo

- Habilidades interpersonales

- Trabajo en equipo interdisciplinario

- Capacidad para comunicarse con expertos de otras áreas

- Reconocimiento de la diversidad y multiculturalidad

- Habilidad para trabajar en un contexto internacional

- Compromiso ético

Sistémicas

- Capacidad de aplicar los conocimientos en la práctica

- Habilidades de investigación

- Aprendizaje

- Adaptación a nuevas situaciones

- Capacidad para generar nuevas ideas (creatividad)

- Liderazgo

- Conocimiento de culturas y costumbres de otros países

- Habilidad para trabajar de forma autónoma

- Diseño y gestión de proyectos

- Iniciativa y espíritu emprendedor

- Motivación por la calidad

- Motivación por la consecución de objetivos

blemas y en la consecuente toma de decisiones, la aplicación de la capacidad analítica y sintética forma parte de sus actividades profesionales diarias. Su unión al razonamiento crítico permitirá que tales profesionales no funcionen como una mera correa de transmisión de órdenes superiores sino como expertos que deciden en función de la aplicación de principios racionales y científicos a situaciones específicas.

- CT interpersonales: también son importantes en la formación universitaria. De éstas la capa- 
cidad de trabajar en equipo es esencial en las profesiones sanitarias donde cualquier proyecto -asistencial o de investigación- supone la necesidad de realizar actividades donde se aprenda la dinámica de un grupo de trabajo, la asignación de tareas y el respeto hacia las opiniones de los otros miembros del equipo.

- CT sistémicas: la mayoría de ellas -como la iniciativa o la creatividad- son inherentes a la juventud, y los profesores sólo debemos encauzarlas y engarzarlas con la capacidad para aprender autónomamente, única garantía para la renovación en el conocimiento del profesional.

Una CT que respalda todos los aspectos de la práctica profesional y hace necesaria su incorporación en los planes de estudio son las habilidades comunicativas. Habilidad que comporta la comunicación con el paciente (transmitir diagnósticos, malas noticias y el manejo de pacientes difíciles) y la científica con otros profesionales (sesión clínica, congresos, etc.).

Si bien la habilidad comunicativa (oral y escrita) se considera ya adquirida cuando los estudiantes alcanzan la universidad, la realidad del día a día muestra otra que incluso persiste después de haber alcanzado el grado. Esto hace necesario fomentar la habilidad comunicativa tanto en su expresión escrita como oral; la escrita, mediante la redacción de documentos donde los estudiantes aprendan a presentar de forma lógica y coherente sus puntos de vista sobre un problema. De forma similar, la comunicación oral es deficitaria en un amplio número de universitarios, poco habituados a hablar en público y explicar sus puntos de vista con un lenguaje adaptado al entorno profesional.

Tradicionalmente, las titulaciones en Ciencias de la Salud han centrado sus enseñanzas en las competencias específicas y se ha obviado la preocupación por la instauración de ciertas competencias transversales a pesar de ser consideradas importantes. En España lo más frecuente es que no se consideren de forma explícita una asignatura determinada y muchos docentes sienten que con el tiempo destinado a las competencias específicas no es posible formar adecuadamente a los estudiantes en CT, además las experiencias institucionales también apuntan en esa dirección. No obstante, se ha señalado que los 'minicongresos' y 'minisimposios' son una buena oportunidad para estimular a los estudiantes en el recorrido de su propio aprendizaje y que puedan desarrollar múltiples CT [5].

En la actualidad, las distintas titulaciones de Ciencias de la Salud de la Universidad Complutense de Madrid (UCM) han iniciado un proceso de reforma curricular siguiendo los nuevos retos y cambios en el proceso enseñanza-aprendizaje del sistema universitario español dentro del marco del proceso de implantación del EEES y, por tanto, centrado en el alumno y en la adquisición de aquellas competencias que se consideran imprescindibles para el ejercicio profesional, lo que requiere la innovación en metodologías docentes y en particular en CT.

En este sentido se enmarca la experiencia que presentamos: las Jornadas Complutenses de Investigación para alumnos de pregrado en Ciencias de la Salud (Jornadas), una actividad académica de libre elección creada para impulsar el trabajo colaborativo entre profesores y alumnos, el desarrollo de habilidades en el ámbito de la investigación y la comunicación científica, fomentando su autoaprendizaje [6]. Iniciativa que comparamos con otras experiencias similares previas.

\section{Sujetos y métodos}

\section{Jornadas complutenses}

Estas jornadas surgieron como propuesta de los máximos responsables académicos de las titulaciones sanitarias de la UCM (Medicina, Farmacia, Odontología, Óptica y Optometría, Enfermería, Fisioterapia, Podología, Terapia Ocupacional y Nutrición Humana), con el respaldo de las respectivas juntas de Facultad o Escuela Universitaria para su desarrollo. Se establecieron con periodicidad anual, su sede, y la organización corresponde cada año a uno de los centros participantes.

La Facultad de Medicina fue la encargada de realizar las primeras jornadas que, con carácter regional, se dedicaron al centenario de la concesión del premio Nobel al Profesor D. Santiago Ramón y Cajal, y tuvieron lugar los días 21 y 22 de abril de 2006. El segundo año se celebraron en la Facultad de Farmacia durante los días 20 y 21 de abril de 2007, ya con carácter nacional, y con el nombre de II Jornadas Complutenses y I Con- 
greso Nacional de investigación para alumnos de pregrado en Ciencias de la Salud.

\section{Organización}

Para mantener una estructura organizativa, se estableció un comité directivo en el que estaban presentes los decanos de facultades y directores de escuelas, un comité organizador local de cada centro operativo durante el curso académico en el que se desarrollaban las Jornadas, y un consejo ejecutivo intercentros, constituido por dos profesores por centro.

En cada Jornada se celebraban sesiones organizadas por afinidad temática donde los alumnos presentaban los trabajos de investigación, que podían ser o bien de búsqueda o actualización bibliográfica o bien de laboratorio, siempre realizados -como condición obligatoria- con la tutorización de un profesor $[7,8]$. La comunicación podía presentarse como modalidad oral o tipo póster. En ambos casos, el alumno tenía que defender su trabajo ante los moderadores asignados a la correspondiente sesión (10 minutos para la oral y 3-4 minutos para los pósteres). No había límite en el número de comunicaciones por alumno.

Junto a las sesiones de presentación de trabajos se programaba una conferencia en la que se invitaba a un investigador que, por su trayectoria profesional, servía de ejemplo para los alumnos y testimonio de la importancia de la investigación en Ciencias de la Salud.

\section{Objetivos}

Los objetivos propuestos de las Jornadas se recogen en la tabla II. El objetivo general era que el alumno se familiarizase con la metodología científica de la investigación, uno de los pilares fundamentales de la formación universitaria y del desarrollo de habilidades comunicativas.

No obstante, y siendo realistas, el elemento esencial de aprendizaje se sigue basando en el eje motivación-recompensa; por ello la participación de los alumnos en las Jornadas es recompensada con el reconocimiento de créditos de libre configuración en función de una serie de variables establecidas en el Reglamento de la UCM para este tipo de actividades. También se estableció un premio simbólico por cada 20 comunicaciones presentadas mediante una valoración ponderada entre los miembros del co-

\section{Tabla Il. Objetivos de las Jornadas Complutenses de Investigación para alumnos en Ciencias de la Salud.}

- Facilitar, a través de la presentación de trabajos, la formación del alumno en habilidades comunicativas

- Familiarizar a los alumnos en la metodología y técnicas de investigación en Ciencias de la Salud

- Hacer partícipe y protagonista al alumno en el campo de la investigación en Ciencias de la Salud, resaltando la importancia que esta faceta tiene en su formación global universitaria

- Incorporar a los alumnos a los grupos de investigación competitivos en la Universidad Complutense y facilitarles el trabajo en equipo

- Alinear las actividades de los alumnos a lo establecido en el Espacio Europeo de Enseñanza Superior

- Implementar las actividades en el campo de la Innovación Educativa

mité intercentros y los moderadores de la mesa. Los primeros valoraban la 'letra' del trabajo y los segundos la 'música' de la presentación, de este modo los moderadores de sesión debían concentrarse en las cualidades comunicativas de la presentación y de quién la presentaba, obviando el posible sesgo que implicaría evaluar comunicaciones de su área de conocimiento.

\section{Gestión}

Para planificar la gestión de las Jornadas, se decidió establecer en el campus virtual de la UCM un espacio web. Desde octubre de 2005 se habilitó un entorno dentro de la opción de Seminarios de Trabajo para presentar las Jornadas en los centros y realizar la difusión entre el profesorado y alumnado, de forma que cuando se editaron los dípticos y carteles anunciadores se incluyó la dirección electrónica y la de los centros participantes con el que estaba enlazado.

\section{Valoración}

El grado de satisfacción de las Jornadas se evaluó anónimamente tanto por los alumnos como por los profesores que actuaron como moderadores en las distintas sesiones.

En la primera edición, se preguntó a los alumnos sobre la utilidad de la experiencia y la rele- 


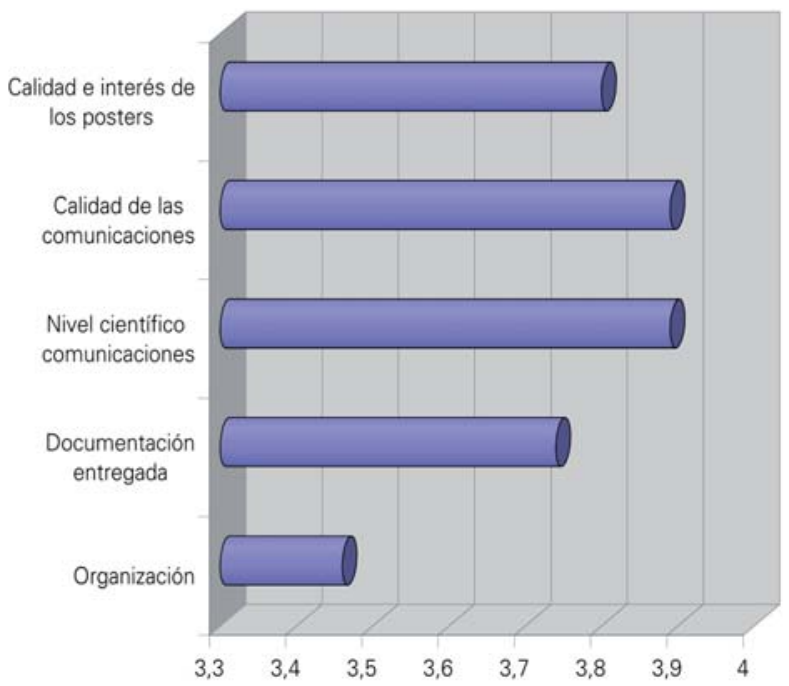

Figura 1. Valoración de las I Jornadas por los alumnos (ponderación: mínima = 0, máxima = 5)

vancia que este tipo de actividad tiene en la formación universitaria, así como en la de su titulación. Se les pidió que valorasen entre 0 y 5 diversos aspectos como la organización, la documentación entregada, el nivel científico de las comunicaciones, la calidad de las comunicaciones y la calidad e interés de los pósteres presentados.

En las II Jornadas se les solicitó que valorasen sobre la trascendencia que este tipo de actividad tiene para ellos según una serie de ítems: aprender metodología científica (metodología), aprender a hablar en público (oratoria), aprender redacción científica (redacción), relevancia en los estudios (relevancia) y obtención de créditos de libre configuración (créditos); entre paréntesis hay la denominación reseñada en la figura 1.

De igual modo, a los profesores moderadores de las II Jornadas se les pidió que valorasen entre 0 y 10 sobre la originalidad, planteamiento metodológico, presentación iconográfica, exposición oral, viabilidad y relevancia de las comunicaciones presentadas (Fig. 2).

\section{Resultados}

En estas dos ediciones se inscribieron un total de 1.434 alumnos (Tabla III). En las I Jornadas se inscribieron 500 alumnos, todos de la UCM, de los cuales 331 participaron simplemente como asistentes. Se presentaron un total de 169 comunicaciones [7] (129 orales, 40 pósteres). En las II Jornadas lo hicieron 934 alumnos, de ellos 838 de la UCM y 96 de otras universidades (Alcalá de Henares, Alfonso X el Sabio, CEU San Pablo, Europea, Granada, Oviedo, Pontificia de Salamanca, Pública de Salamanca y Santiago de Compostela); de todos ellos 648 participaron en calidad sólo de asistentes. Se presentaron un total de 286 comunicaciones [8] (180 orales, 106 pósteres). La tabla III proporciona más detalles al respecto.

Para las I Jornadas se constituyeron 21 mesas de comunicaciones y en las segundas un total de 35, en cada una de las cuales había dos moderadores y se presentaban de seis a siete comunicaciones. Respecto a las sesiones de póster hubo cuatro sesiones en la primera edición y tres en las II Jornadas.

Sólo un 5\% de alumnos participantes en las I Jornadas lo había hecho con anterioridad en otros eventos similares. En cuanto a la valoración por parte de los alumnos (n. ${ }^{\circ}$ de respuestas), un 99\% consideró que este tipo de actividades era relevante en la formación de su titulación, y un 98\% que este tipo de actividades era relevante en su formación universitaria. 


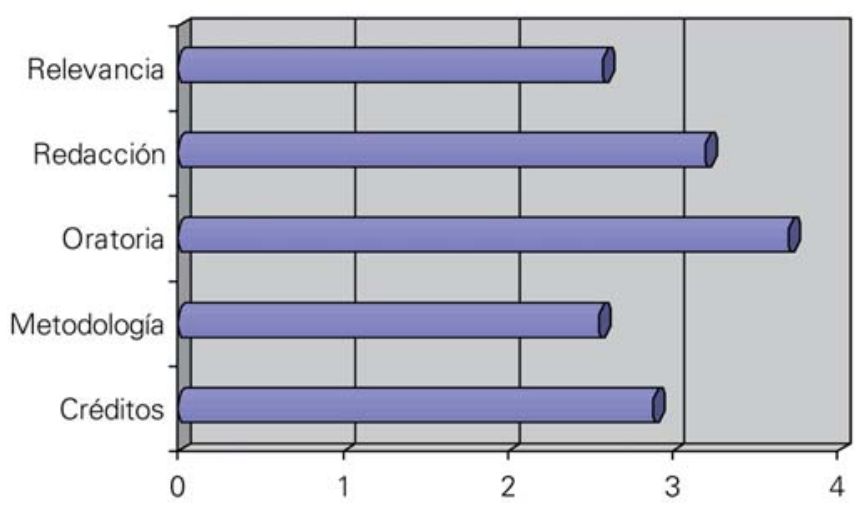

Figura 2. Valoración del interés de las II Jornadas por los alumnos (ponderación: mínima =0, máxima = 5).

Al 100\% de los alumnos de las dos ediciones les parecieron interesantes las Jornadas.

En las I Jornadas (Fig. 1), las mayores valoraciones fueron para la calidad de las comunicaciones $(3,89)$ y el nivel científico de éstas $(3,89)$, seguido de la calidad y el interés de los pósteres $(3,8)$; la menos valorada fue la organización $(3,46)$. En las II Jornadas se les preguntó que ordenasen de 1 (valor mínimo) a 5 (valor máximo) la trascendencia que para ellos tenía este tipo de actividad, según los ítems referenciados (Fig. 1): aprender a hablar en público (oratoria) fue el más valorado $(3,7)$, seguido de aprender redacción científica (redacción) $(3,2)$, la obtención de créditos (créditos) $(2,88)$, relevancia en los estudios (relevancia) $(2,58)$ y, en último lugar, aprender metodología científica (metodología) $(2,55)$.

Respecto a la valoración de los moderadores de la mesa -68 respuestas- (Fig. 2), la máxima calificación fue la relevancia de la actividad $(9,7)$, seguido de la presentación iconográfica $(8,41)$, la exposición oral $(8,26)$ y la viabilidad de las comunicaciones presentadas $(8,11)$; las menos valoradas fueron la originalidad $(7,46)$ y el planteamiento metodológico $(7,44)$.

\section{Discusión}

La excelencia ha sido siempre un objetivo de la institución universitaria. En la actualidad se entiende la formación de profesionales en términos de competencia, es decir, del conjunto de conocimientos, habilidades y actitudes que permiten una práctica profesional excelente en continuo perfeccionamiento y adecuada al contexto social en que se desarrolla. Hoy no podemos asegurar la excelencia para todos nuestros alumnos, pero sí la igualdad de oportunidades, y a esto contribuyen estas Jornadas.

Para la mayoría de los alumnos la participación en estas Jornadas supone un primer contacto para aprender cómo comunicar en el ámbito de la ciencia, una habilidad que por lo referenciado anteriormente creemos muy útil para su futuro profesional. Es una actividad que capta y estimula el interés del alumno. Mediante la presentación de los trabajos, se consigue que el alumno desarrolle habilidades y aptitudes en la comunicación oral, y se inicia en el proceso de difusión científica de los resultados. Se convierte, de esta forma, en el protagonista de su formación, mejora su itinerario formativo, participa con el desarrollo de actividades en el campo de la innovación educativa, mejora en el uso de las nuevas tecnologías, y trabaja conforme a la filosofía del EEES. De este modo, el alumno pasa a ser un gestor activo [6] y difusor de su aprendizaje, y garantiza la consecución de un perfil de graduado acorde con la demanda actual de la sociedad.

La iniciación en el proceso de investigación al alumno universitario, en general y en particular, de cualquier titulación de Ciencias de la Salud, desde el comienzo de sus estudios universitarios adquiere cada vez más importancia en el 
Tabla III. Alumnos inscritos y tipo de participación clasificados por titulaciones.

\begin{tabular}{|c|c|c|c|c|c|c|}
\hline & \multicolumn{3}{|c|}{ Curso 2005-2006 [7] } & \multicolumn{3}{|c|}{ Curso 2006-2007 [8] } \\
\hline & Inscritos & Oral & Póster & Inscritos & Oral & Póster \\
\hline Biología & 11 & 2 & 0 & 16 & 3 & 1 \\
\hline Bioquímica & 0 & 0 & 0 & 1 & 0 & 0 \\
\hline Biotecnología & 0 & 0 & 0 & 2 & 1 & 0 \\
\hline $\begin{array}{l}\text { Ciencia y Tecnología } \\
\text { de los Alimentos }\end{array}$ & 1 & 1 & 0 & 2 & 2 & 1 \\
\hline $\begin{array}{l}\text { Dirección y Administración } \\
\text { de Empresas }\end{array}$ & 1 & 0 & 0 & 1 & 0 & 0 \\
\hline Enfermería & 8 & 1 & 1 & 44 & 8 & 1 \\
\hline Farmacia & 88 & 22 & 9 & 371 & 58 & 47 \\
\hline Física & 0 & 0 & 0 & 1 & 0 & 1 \\
\hline Fisioterapia & 45 & 16 & 1 & 33 & 10 & 0 \\
\hline Físicas & 0 & 0 & 0 & 0 & 0 & 1 \\
\hline Logopedia & 0 & 0 & 0 & 1 & 0 & 0 \\
\hline Magisterio & 0 & 0 & 0 & 2 & 0 & 0 \\
\hline Medicina & 112 & 47 & 9 & 107 & 39 & 15 \\
\hline $\begin{array}{l}\text { Nutrición Humana } \\
\text { y Dietética }\end{array}$ & 26 & 6 & 0 & 89 & 18 & 12 \\
\hline Odontología & 39 & 7 & 5 & 21 & 11 & 0 \\
\hline Óptica y Optometría & 89 & 3 & 3 & 85 & 6 & 5 \\
\hline Periodismo & 0 & 0 & 0 & 1 & 0 & 0 \\
\hline Podología & 27 & 6 & 3 & 25 & 7 & 5 \\
\hline Psicología & 0 & 0 & 0 & 1 & 0 & 0 \\
\hline Químicas & 0 & 0 & 0 & 3 & 0 & 0 \\
\hline Terapia Ocupacional & 51 & 17 & 9 & 124 & 19 & 17 \\
\hline Traducción & 1 & 0 & 0 & 0 & 0 & 0 \\
\hline Turismo & 0 & 0 & 0 & 1 & 0 & 0 \\
\hline Veterinaria & 1 & 1 & 0 & 3 & 0 & 0 \\
\hline Total & 500 & 129 & 40 & 934 & 180 & 106 \\
\hline
\end{tabular}




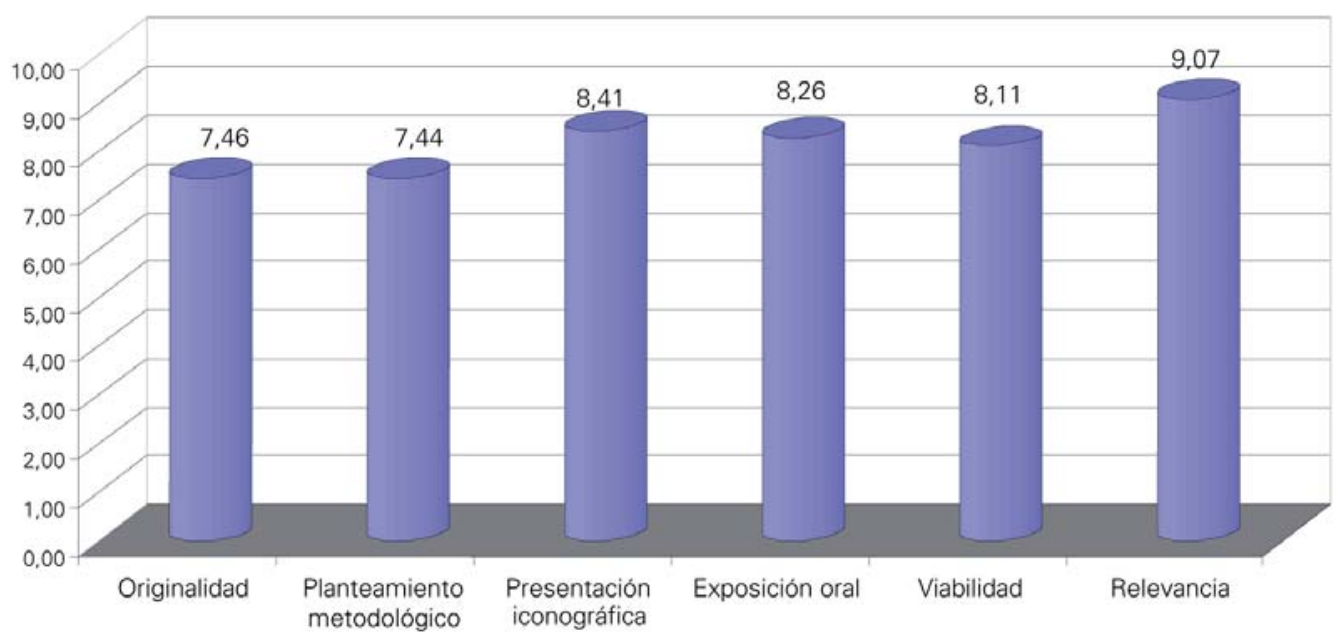

Figura 3. Valoración de las comunicaciones de las II Jornadas por parte de los moderadores (ponderación: mínima =0, máxima = 10).

contexto del EEES. Si se consigue que el alumno participe de forma activa en este proceso, es muy posible que desarrolle de forma temprana habilidades comunicativas, una mayor vocación científica; que modele una voluntad eficaz y metódica como exige y precisa la ciencia [9].

Para ese fin, puede ser interesante el establecimiento de asignaturas específicas en metodologías de investigación en Ciencias de la Salud en la que los futuros sanitarios, bajo la tutorización de profesores universitarios y con el empleo de un sistema mixto de aprendizaje basado en el componente virtual, tienen que realizar un trabajo de investigación y presentarlo en alguno de los congresos de pregrado concebidos para ese fin, como pueden ser las Jornadas, que en la actualidad ven incrementado tanto su número de inscripciones como el volumen de participantes activos, dado el reconocimiento y el valor que están consiguiendo en estos últimos años.

Pero el éxito de este tipo de actividades no sólo depende de la participación de los alumnos, sino también de la implicación de los profesores, que orientan a los alumnos en la realización de sus trabajos o participan en la logística de la misma. Esto proporciona una medida ponderable que puede contribuir o considerarse como dato objetivo en la evaluación docente del profesorado.
Una característica diferencial de las Jornadas Complutenses respecto a otras actividades similares [5] es que no se limita a estudiantes de una misma titulación o área de conocimiento específica, todos ellos con una lógica y marcada verticalidad en contenidos, sino que, por el contrario, su vocación es ser tangencial a ellos $\mathrm{y}$ abiertas a cualquier estudiante universitario siempre que su trabajo esté enfocado a algún aspecto relacionado con la salud. Ello proporciona no sólo formación e información, sino que lo convierte en un verdadero punto de encuentro multidisciplinar que puede permitir al alumno desarrollar una actitud abierta, activa, crítica y constructiva, tomando como referencia las Ciencias de la Salud.

Este tipo de actividad proporciona beneficio no sólo al alumno que participa sino también a los profesores y a la institución. En nuestra experiencia, gracias a su relación con el alumno tutorizado los profesores obtienen un mayor reconocimiento de su estatus profesional al igual que la institución universitaria que la fomenta. De este modo, las Jornadas tienen un valor formativo e informativo para el alumno, el profesor y la institución universitaria que las convierten en una 'terapia pedagógica holística', por lo que las consideramos una experiencia positiva. 


\section{Bibliografía}

1. Real Academia Española de la Lengua. Diccionario de la Lengua española. 22 ed. Madrid: Espasa; 2001. p. 604.

2. Barón M. La enseñanza de la medicina. In Villanueva JL, Millán J, Barón M, eds. Estándares para la enseñanza de la medicina. Madrid: Fundación Lilly; 2006. p. 7-8.

3. Baños JE, Pérez J. Cómo fomentar las competencias transversales en los estudios de Ciencias de la Salud: una propuesta de actividades. Educ Med 2005; 8: 216-25.

4. González J, Wagenaar R. Tunning educational structures in Europe. Informe final. Deusto: Universidad de Deusto; 2003.

5. Tamayo G, Santibáñez M, Meana JJ. De la enseñanza tradicional al aprendizaje basado en el desarrollo de un proyecto de investigación. Una experiencia de transición en Farmacología. Educ Med 2000; 8: 40-9.
6. Smith J. Practice for lifelong learning through independent learning. In Bourner T, Katz T, Watson D, eds. New directions in professional higher education. Philadelphia: Open University Press; 2000. p. 256.

7. I Jornadas Complutenses de investigación de alumnos de pregrado en Ciencias de la Salud, programa y resúmenes. Facultad de Medicina, 21-22 de abril de 2006. Madrid: ADEMAS Comunicación. p. 191.

8. II Jornadas Complutenses y I Congreso Nacional de investigación para alumnos de pregrado en Ciencias de la Salud, programa y resúmenes. Facultad de Farmacia. 2021 de abril de 2007. Madrid: Gráficas San Marcos. p. 332.

9. Gracia-Guillén D. El otro Cajal. In Facultad de Medicina, ed. Santiago Ramón y Cajal: un Nobel Complutense. Madrid: Universidad Complutense; 2007. p. 34. 